\title{
Design and Verification of Application Specific Integrated Circuits in a Network of Online Labs
}

\author{
Danilo Garbi Zutin ${ }^{1}$, Michael E. Auer ${ }^{1}$ and A. Y. Al-Zoubi ${ }^{2}$ \\ ${ }^{1}$ Carinthia University of Applied Sciences, Villach, Austria \\ ${ }^{2}$ Princess Sumaya University for Technology, Amman, Jordan
}

\begin{abstract}
A solution to implement a remote laboratory for testing and designing analog Application-Specific Integrated Circuits of the type (ispPAC10) is presented. The application allows electrical engineering students to access and perform measurements and conduct analog electronics experiments over the internet. PAC-Designer software, running on a Citrix server, is used in the circuit design in which the signals are generated and the responses are acquired by a data acquisition board controlled by LabVIEW. Three interconnected remote labs located in three different continents will be implementing the proposed system
\end{abstract}

Index Terms-Online Laboratories, e-Learning, Virtual Instrumentation,

\section{INTRODUCTION}

Technology-enhanced learning is becoming a new important trend in higher education worldwide. In particular, engineering education is becoming an exciting emerging field of research because it involves a multitude of disciplines which aim to resolve the pedagogical problems that arise with the advancement of technology. In addition, laboratory experiments and instruments are becoming sophisticated and expensive for universities to purchase and maintain. Remote labs offer a solution and represent a practical alternative through which students may conduct experiments online, anywhere and at anytime. Compared with traditional laboratory practice, remote labs offer flexible learning in time and place, access to a wide number of distributed experiments and cost-cutting strategies 1-4.

Nevertheless, it is still difficult to share instrumentation and experiments among laboratories [3]. Each one has its own security policy and adopts a proper technology in accessing and controlling real devices. A common integrated framework, offering indexing facilities, unique logins, file sharing and the seamless access and run of experiments, is the main challenge in order to create a network of online laboratories. Grid technologies can be used to set up an effective network of remote laboratories for education purposes by sharing instrumentation and resources. However, the evolution of remote laboratories from the current client/server architecture to grid-based architecture requires well-defined tools for location, security, and integration of resources, and further research is currently being conducted to examine this issue [5-7].

In the meantime, the goal of developing a tool for creating remote experiments remains essential to provide teachers with resources for designing analog electronics experiments, which may involve controlling the gain of an amplifier or designing different types of filters and activating relays, electronic switches, among other devices, without concern to the problems related to programming instruments, hardware communication and their integration to the Internet, being able to focus on the aspects related to the subject they want to explore.

In this paper, an analog electronics interactive laboratory (iLab) is developed, at Princess Sumaya University for Technology, Jordan, as an attempt to enable students to perform remotely and in real time several distinct experiments. The laboratory will form part of a network of web-based remote labs as efforts; by the Remote Electronic Lab (REL), Carinthia University of Applied Sciences, Austria are continued in this direction [8-9]. The laboratory is based on an Application-Specific Integrated Circuits, of the type (ispPAC10), which can be programmed by PAC-Designer software. The signals are generated and the responses are acquired by a data acquisition board controlled by LabVIEW. The ultimate aim is to link REL with iLab, and the remote lab at Universidade Estadual Paulista, Brazil.

Simple experiments were performed within the range of the ispPAC10 analog functions. As examples can be said a $20 \mathrm{~dB}$ gain amplifier and analog low-pass, high-pass and band-pass filters. The cutoff frequencies can be adjusted by the user based on the ispPAC 10 adjustable parameters. Basically, other analog functions can be implemented, depending on the user's wish.

\section{A. Configuration of the ispPAC 10}

The ispPAC10 is an in-system programmable analog device from Lattice Semiconductor, which allows the implementation of several analog circuits such as low-pass and band-pass filters, amplifiers, oscillators, etc. Its basic building block contains four integrated programmable analog modules known as PACblocks and a programmable analog routing pool, as shown in Fig. (1). Each PACblock emulates a collection of op amps, resistors and capacitors. The ispPAC10's flexibility permits one to vary a filter's gain, corner frequency, and other characteristics without the need for external components. Additionally, because the ispPAC10's analog circuitry is based on continuous-time, as opposed to switched-capacitor technology, filters implemented with the ispPAC10 are not subject to the sampling and aliasing problems often encountered when using switched capacitor filters. Due to the frequencies used for our experiments we understand that the sampling/aliasing problems associated with switched capacitors are far from being noticed, although this issue describes one feature of 
the ispPAC device that might be important depending on the application developed with it. The ability to program the internal capacitor values of the ispPAC10 allows the designer to realize thousands of distinct analog circuits and filter characteristics from a given circuit architecture.

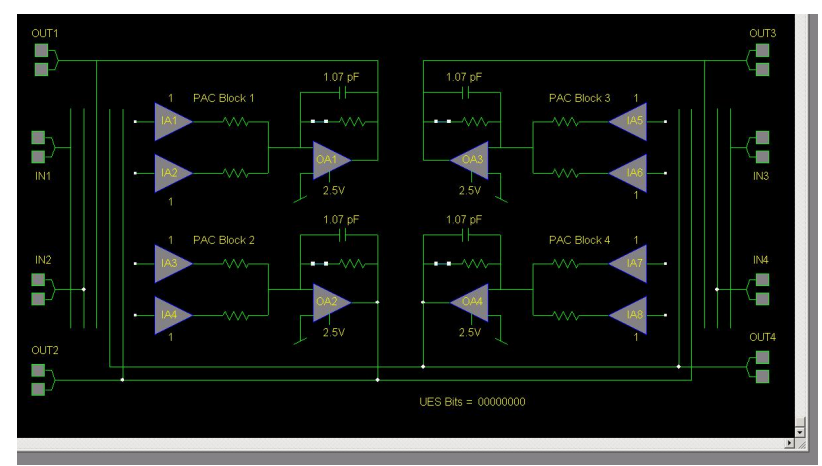

Figure 1. Basic Building Block of the ispPAC10.

By assuming a fixed architecture, the ordinarily complex task of designing a desired analog circuit or filter can be reduced to a simple, table-based process requiring minimal calculation. The ispPAC10's in-system programmability enables programming, verification, and reconfiguration when the device is assembled onto a printed circuit board using an IEEE standard 1149.1 compliant serial port (JTAG). Circuit programming and uploading are achieved by means of a windows-based application called PAC-Designer. The analog circuit can be design and simulated at a PC with free schematic-based software and a built-in simulator or it can be exported to PSPICE and instantly downloaded to configure an E2programmable Lattice ispPAC.

A block diagram of the remote lab ispPAC10 analog circuit is shown in Fig. (2), in which the programmed chip is connected to a National Instruments LabVIEWcontrolled data acquisition board (DAQ/USB-6251).

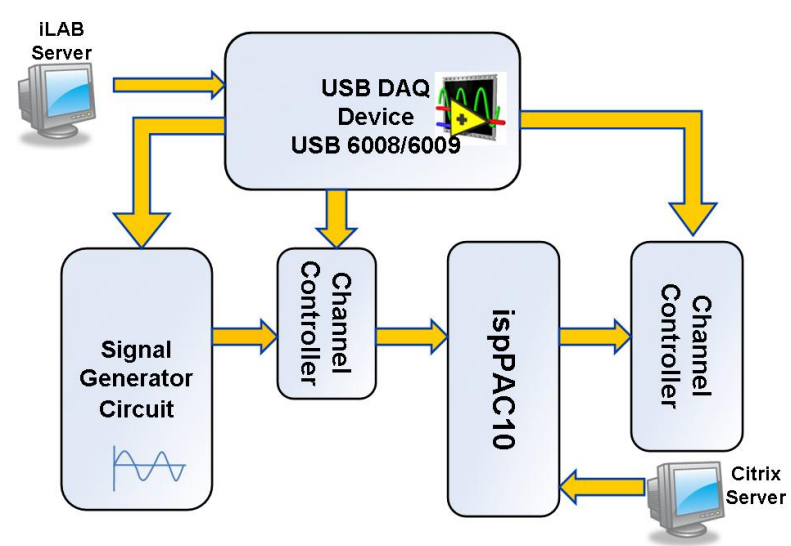

Figure 2. The Remote Lab ispPAC10 Analog Circuit.

The signal input to the circuit can be generated internally, with the DAQ card or externally using a signal generator. The circuit was designed and assembled to offer the same functionalities to the remote lab user. Furthermore, this remote analog electronics laboratory may also be used for testing and designing analog application specific integrated circuits other than the ispPac10 such as ispPac20, ispPac80 and similar chips.

The ispPAC10 should be first configured in such a way as to connect the differential inputs to a single-ended signal because the device is fully differential. Thus, one of the differential inputs, VREFOUT, was connected to a DC bias of $2.5 \mathrm{~V}$. Another important concern is that only one user can connect to the system at a time.

The input signal must either be AC coupled or have a DC bias equal to the DC level of the other input. The ispPAC10 operates within a voltage range from 1 to $4 \mathrm{~V}$. Once all performed experiments were carried out in single ended mode, all the inverter analog inputs were connected to VREFOUT $(2.5 \mathrm{~V})$. Due to the hardware limitations, the function generator could not generate signals within a large bandwidth, although the circuit behaviors could be well visualized for the performed exercises. At the CTI a DAQ card USB 6251 from National Instruments was used. This device has an input sampling rate of $1.25 \mathrm{MS} / \mathrm{s}$ and an output sampling rate of 2.86 MS/s. We considered a minimum of 20 samples per period of the generated signal, which leads to a maximum frequency of $143 \mathrm{KHz}$ possible for our analog waveform generation. For the input, the sampling rate is divided by 2 as two analog channels are measured simultaneously (the DAQ card has only one ADC).

Another important aspect found after the board development is the noise level that affects the measurements performed in the circuit. For signals with low amplitude and high frequencies the noise effects could be well noticed. For higher amplitudes this effect could badly be noticed. Although it is an important consideration because the experiments with the ispPAC10 device can only be performed within a voltage range from 1 to $4 \mathrm{~V}$, which leads to the constant use of low amplitude signals when conducting experiments. No measurements though were carried out to quantify the noise (white noise) level, but the presence of noise could be noticed on the waveform signals when performing experiments with low amplitude levels $(0,1$ to $0,2 \mathrm{~V})$. In order to solve that problem a cable with individually shielded analog twisted pairs was used.

\section{SYSTEM DESIGN}

The remote electronic laboratory system has the architecture shown in Fig. (3). Real experiments are carried out on the server side, where the user can control these experiments via the Internet by setting the parameters of the input signal such as waveform, frequency, amplitude, offset. The circuit outputs may then be read and compare with the input waveform signal.

The user interface and the control of the lab hardware were developed with LabVIEW. Only one user can access the remote lab at a time, therefore the user control access system a thirty minutes time-slot for each user connected to the system. To access the remote lab, the user is asked to insert a username and a password. If both are valid, the system enables the access to the lab, as shown in Fig. (4).

The basic operation of the remote lab was based on controlling the DAQ board by programming LabVIEW virtual instruments (VI) as well as the sub VIs. The virtual lab equipments such as the function generator and oscilloscope were created also using LabVIEW.A web server hosts the HTML files that contain the ASIC laboratory home page with information about the implemented exercises, as well as the links to login on the system and it is located, in our case, apart from the LabView server. 


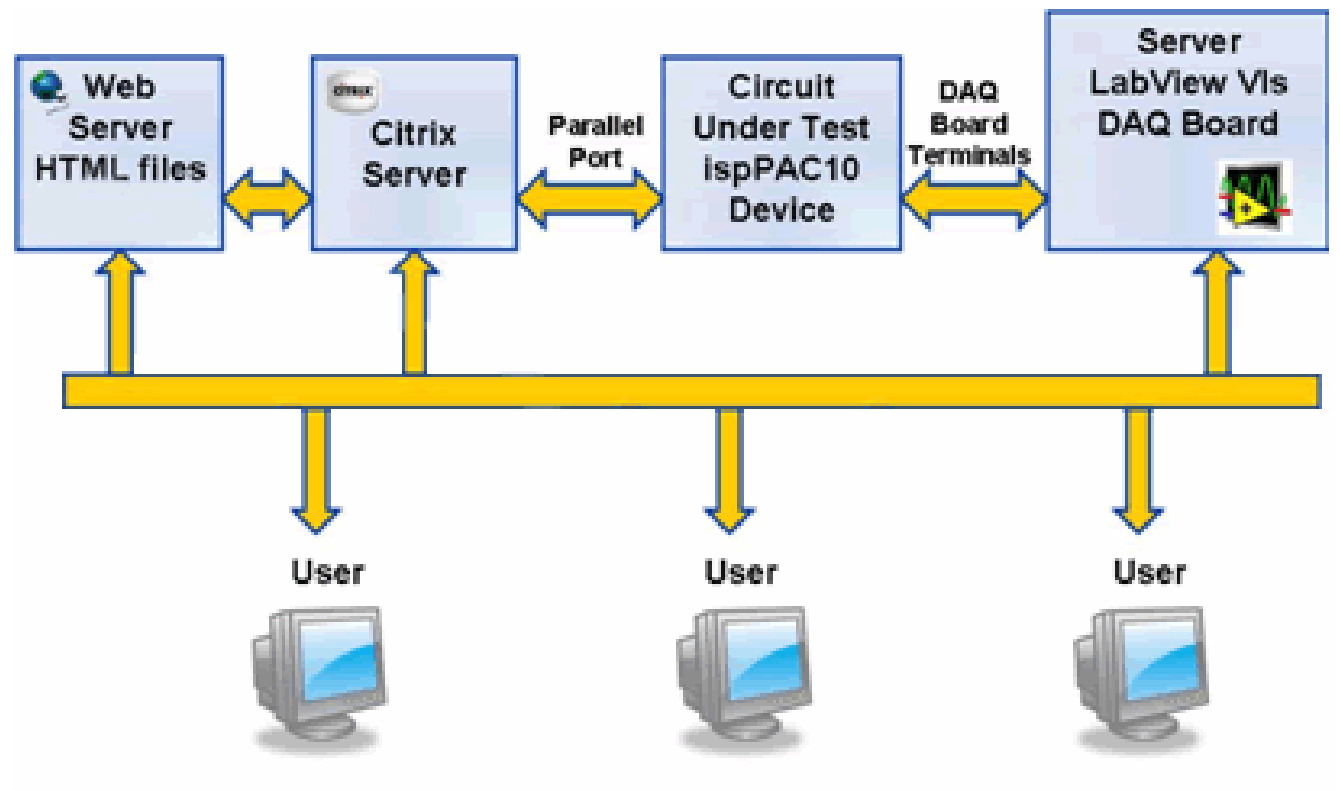

Figure 3. The Remote Electronic Laboratory System.

The LabView server is connected to the circuit under test via a DAQ (Data Acquisition) card and therefore controls all the data acquisition and generation for the experiments, as well as all the measurements performed. The data acquisition device used for this application is the USB 6251, from National Instruments. This device offers two analog outputs and 16 analog inputs, with sampling rates of $1.25 \mathrm{MS} / \mathrm{s}$ and $2.86 \mathrm{MS} / \mathrm{s}$ respectively. This DAQ card also offers 24 digital input/output lines.

The driver software has an application programming interface (API), which is a library of VIs, functions, classes, attributes, and properties for creating applications. This is a library of polymorphic VIs that replaces large parts of a complex DAQ application with a simple configuration dialog.

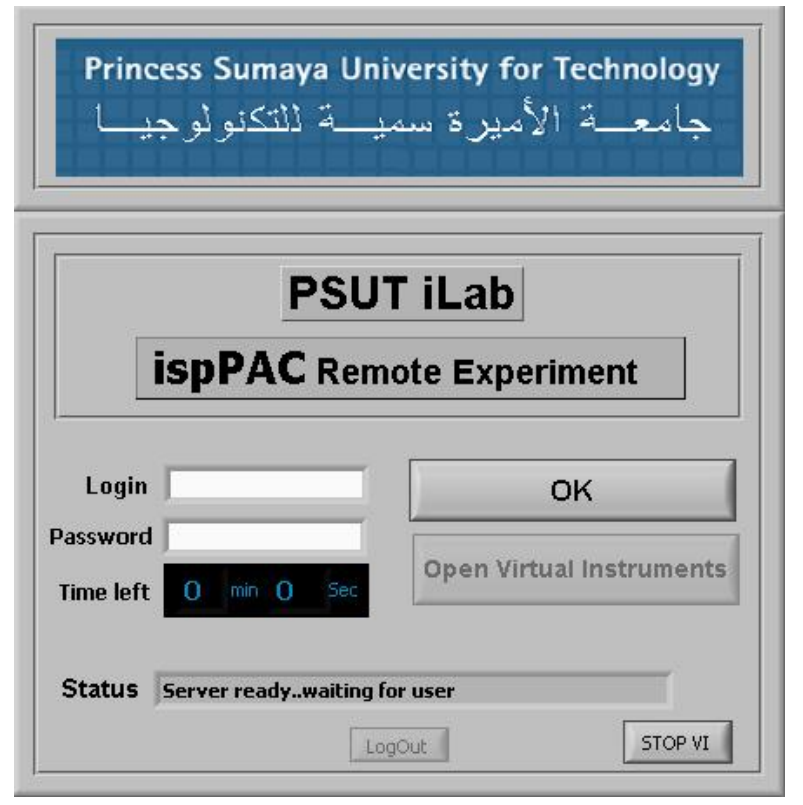

Figure 4. A Front Panel View of the Remote Access.
The development of the virtual instruments can be divided into three parts, the virtual oscilloscope, the virtual function generator and the user access control, each one with well defined tasks. The virtual oscilloscope should work similarly to a real oscilloscope. The user must be able to measure some properties of the signal and also to visualize the waveform signals. In order to do that, the VIs acquires data from two analog channels simultaneously. The data acquisition properties such as sampling rate and number of samples are calculated based on the parameter called TimeBase sent by the user according to the following equation:

TimeBase $\times$ Sampling Rate $=$ No. of samples $(1)$

This parameter specifies the time interval to be displayed on the oscilloscope screen. The calculation is an iterative process. On each iteration, the sampling rate is decreased by one unit and the number of samples is calculated. This process is repeated until the number of samples reaches a maximum value. This calculation is necessary to prevent a misrepresentation of high frequency signals and to ensure that the number of samples will be enough to visualize the requested time interval when sampling lower frequencies signals.

The ispPAC10 device has 4 analog inputs and 4 analog outputs and for each input/output was assigned an analog input of the DAQ board, so that each one might be read. The user can choose which pair input/output will be displayed by setting the proper parameters. The USB6251 has an input sampling rate of $1.25 \mathrm{MS} / \mathrm{s}$, but the hardware has only one ADC (Analog to Digital Converter), which leads to a rate of $625 \mathrm{KS} / \mathrm{s}$ for each channel. The oscilloscope VI also performs measurements (Function OUT) after acquiring the samples. These measurements which can be accomplished using some VIs from the LabView libraries are Vpp, Vmax, Vmin, Vrms, Vaverage, frequency and period. 
The function generator VI uses the DAQmx API VIs to output an analog waveform signal. The user is able to choose the waveform shape, the amplitude, the frequency and the signal offset. The DAQ board USB-6251 has 2 analog output channels that support a sampling rate of 2.8 $\mathrm{MS} / \mathrm{s}$. In order to control the waveform generation, important parameters are the number of samples per buffer and the sampling rate. Although, these parameters should not be of the users concern, so, LabVIEW calculates these parameters based on the desired frequency provided by the user and ensures the number of samples to be as grater as possible, thus keeping a good quality of the signal. This is achieved according to the following equation:

Frequency $\times$ No. of samples per period $=$ Sampling rate

As he DAQ card used (NI USB 6251) has only 2 analog outputs and the ispPAC10 device has 4 inputs, we assumed that the user should be able to choose to which input apply signals. Therefore an analog 3x8 MUX (only 4 outputs connected) was used.

Concerning the data acquisition, the DAQ card has 16 analog inputs, which allow 8 analog channels to be read simultaneously (differential mode). We assumed that the user should be able to read and analyze the signals applied to the ispPAC10 inputs and outputs. As there are 4 inputs and 4 outputs, there are 8 different nodes that the user should be able to measure. Therefore there is no need for an analog MUX. A data acquisition of two simultaneous channels was configured in order to read one input and one output of the ispPAC10 at the same time.

The user interfaces of the system are the LabView front panels of the virtual instruments. In order to make these virtual instruments available for remote users, the VIs front panels were embedded into HTML with LabVIEW and were published with its built in Web server. Therefore, the front panels are remotely reachable via a standard web browser and they were designed in a way that the user doesn't need any knowledge in LabView to take advantages of the system facilities. Therefore, the client simply needs a web browser and the proper version of LabView Run-time Engine that contains some libraries and web browses' plug-ins. This software is available free of costs at the National Instruments website and it is automatically downloaded on the first attempt to access the system.

The PAC-Designer software was installed on a Citrix server. The Citrix MetaFrame is a Windows NT application for delivering Windows based applications to several desktops and supports up to 15 simultaneous connections. Once installed, the PAC-Designer becomes available to be remotely accessed. The user access system does not control the access to the Citrix server, which must be done separately.

The remote lab hardware incorporating the ispPAC10 circuitry running at the PSUT (Amman, Jordan) is shown in Fig. (5). This system uses a USB 6009 DAQ card and an external circuit to generate signals because this hardware has a much more limited sampling rate (48 $\mathrm{KS} / \mathrm{s})$. The user control access interface of the experiment input/output is shown Fig. (6).

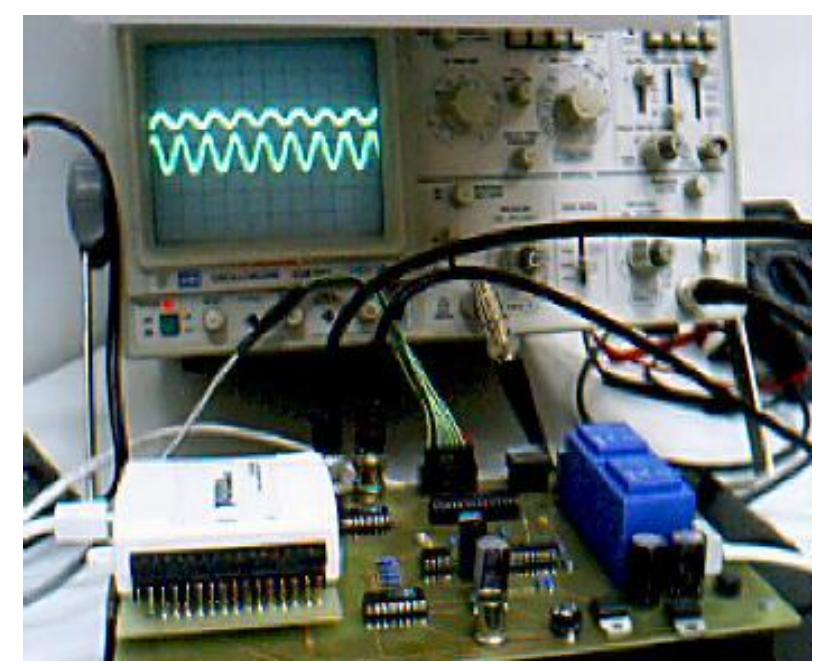

Figure 5. The iLab ispPAC10 Analog Circuit Board, showing the NI DAQ card.

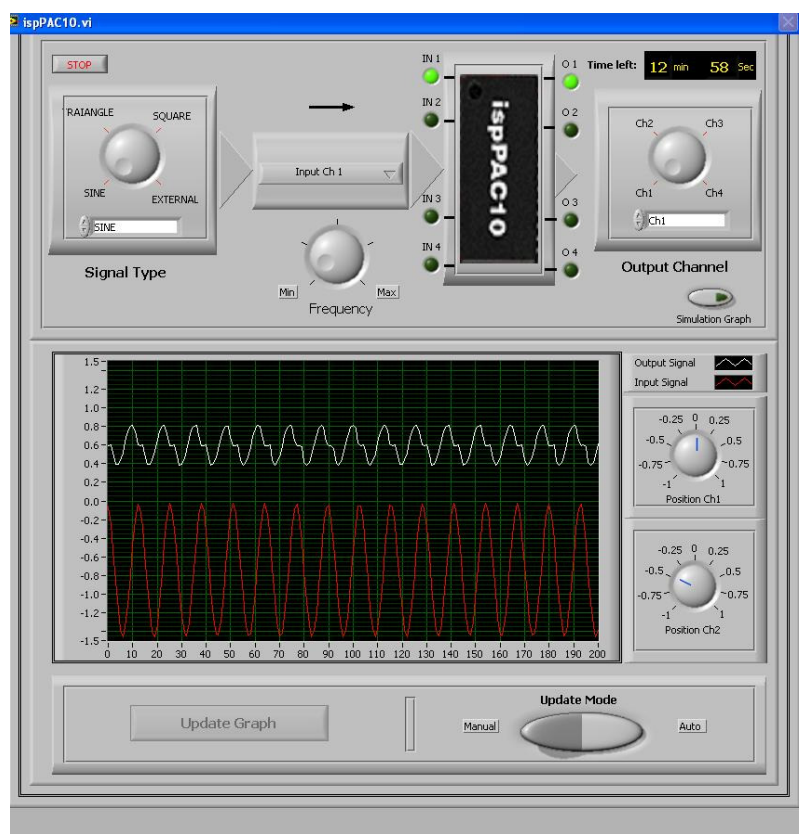

Figure 6. The ispPAC Experiment Input/Output.

\section{CONCLUSION}

A remote analog electronics lab was successfully designed and implemented based on analog ApplicationSpecific Integrated Circuits of the type ispPAC10. The PAC-Designer software is utilized to program the chip. It allows users to create circuit schematics, upload the design and subsequently perform measurements on the real hardware implementation. Two separate systems were developed with two different DAQ cards. The software programming was basically the same, but one of them required the signal inputs to the ispPAC10 IC to be generated with the aid of an external circuit. The response of the configured analog circuit was acquired by the data acquisition board in the same manner for both systems. One is running at PSUT and the other one at the CTI. The user interfaces at the client side were made of virtual instruments front panels published on the Web by a LabVIEW publishing tool. It embeds front panels into HTML files to be accessed via standard web browsers. 
Several experiments with the ispPAC device were performed in a satisfactory manner and the prospect of its utilization in advanced application seems promising. In addition, this remote lab solution offers a wide band of possibilities for applications in industries and for eLearning purposes. Three interconnected remote labs located in Austria, Jordan and Brazil will be implementing the proposed system in the near future.

\section{REFERENCES}

[1] Tatsuya Kikuchi, Takashi Kenjo and Shuichi Fukuda, "Remote Laboratory for a Brushless DC Motor", IEEE Transactions on Education, Vol. 44, No. 2, p 207, May 2001. (doi:10.1109/13.92 5859)

[2] Chi Chung Ko, Ben M. Chen, Shaoyan Hu, Vikram Ramakrishnan, Chang Dong Cheng, Yuan Zhuang, and Jianping Chen, "A Web-Based Virtual Laboratory on a Frequency Modulation Experiment”, IEEE Transactions on Systems, Man, and Cybernetics-Part C: Applications and Reviews, Vol. 31, No. 3, pp 295-303, August 2001. (doi:10.1109/5326.971657)

[3] P. Daponte, D. Grimaldi and M. Marinov, "Real-Time Measurement and Control of an Industrial System Over a Standard Network: Implementation of a Prototype for Educational Purposes”, IEEE Transactions on Instrumentation and Measurement, Vol. 51, No. 5, pp 1-8, October 2002. (doi:10.1109/TIM.2002.807808)

[4] G. Canforaa, P. Daponte and S. Rapuanob, "Remotely Accessible Laboratory for Electronic Measurement Teaching", Computer Standards and Interfaces, Vol. 26, pp 489-499, 2004. (doi:10.1016/j.csi.2004.03.001)
[5] 5. Sam Lee and Mayur R. Mehta, "Establishing a Remote Lab for Teaching Enterprise Application Development”, Information Systems Education Journal, Vol. 4, No. 50, pp 1-7, August 8, 2006.

[6] James E. Corter, Jeffrey V. Nickerson, Sven K. Esche, Constantin Chassapis, "Remote Versus Hands-On Labs: A Comparative Study”, 34th ASEE/IEEE Frontiers in Education Conference, Session F1G, 20-23 October 2004, Savannah, GA, USA.

[7] Qin Shuren, Bo Lin and Liu Xiaofeng, "Development of the Networked Virtual Instrument Lab for Vibration Measuring Based on Microsoft.Net”, Instrumentation and Measurement Technology Conference, IMTC 2004, Como, Italy, 18-20 May 2004.

\section{AUTHORS}

M. E. Auer (m.auer@ieee.org) is with the Carinthia University of Applied Sciences and Head of the Center of Competence "Online Labs and E-Leaning, Villach, A9500 Austria (e-mail:).

A. Y. Al-Zoubi (zoubi@psut.edu.jo) is with the Princess Sumaya University for Technology and Dean of Graduate School and Scientific Research , Amman, Jordan.

D. G. Zutin (D.GarbiZutin@ fh-kaernten.at) is with the Carinthia University of Applied Sciences, Villach, Kaernten A-9500 Austria.

Manuscript received 11 October 2008. Published as submitted by the authors. 